\title{
La proximité et la distance dans l'Utopie de Thomas More
}

\author{
ADÈLE CHENÉ
}

Dans une lettre adressée à Erasme en décembre 1516, Thomas More se réjouit de la critique élogieuse de Tunstal au sujet de l'Utopie et il ajoute: "Vous n'avez pas d'idée comme je me réjouis, comme je suis devenu grand et comme je tiens haut la tête, quand j'imagine que mes Utopiens m'ont choisi pour être leur roi pour toujours. J'imagine que je m'avance, couronné d'un diadème de blé, cet insigne, très impressionnant avec mon froc de franciscain et portant, comme sceptre imposant, une tige de blé. Je suis entouré d'une délégation distinguée d'Amaurautains et je vais au devant des ambassadeurs avec leur suite nombreuse et des souverains étrangers, ces pauvres créatures, en comparaison avec nous..."' Thomas More s'incère donc dans la fiction de l'Utopie en s'identifiant en quelque sorte à ses hommes. Dans sa vision, il se rapproche d'un ailleurs qu'il a imaginé, loin des caprices des princes de son époque et ceux-ci devenant les étrangers, et il se trouve à la tête de ce royaume de l'Utopie où les signes de grandeur sont les plus simples pour ces hommes qui ont atteint une perfection non entravée par l'histoire ou la liberté.

Si nous cherchons à faire le voyage qui sépare l'Angleterre du début du XVIe siècle et l'Utopie, si nous tentons d'établir un pont entre la réalité historique et l'imaginaire, nous sommes saisis par l'étonnant pouvoir de distanciation du discours de l'Utopie. Avec le rêve utopique, nous sommes projetés dans un lieu fictif où la réalité est recomposée et perd, à cause d'un déplacement, toute cohérence topique ou historique. ${ }^{2}$ Les analogies sont perturbées et nous nous retrouvons au croisement de lieux et de temps différents, dans un non-lieu et un non-temps impossibles à situer. Sitôt aperçus, les tableaux de l'Utopie s'annulent, car ils sont autres, toujours ailleurs, comme la totalité de l'Utopie est à distance de la réalité historique et dissimule son sens dans un non-sens.

Sans vouloir abîmer le cours fantaisiste de l'Utopie - n'a-t-on pas dit qu'elle était un jeu d'esprit ${ }^{3}$ - nous avons choisi d'en situer les éléments

Cet article est la version révisée d'une communication présentée au VIe Colloque de l'Institut d'études médiévales de l'Université de Montréal 
selon le projet d'un discours de distanciation. Dans le dialogue entre les protagonistes Raphaël Hythlodée, spécialiste du non-sens, et Thomas More lui-même, nous trouvons un support à cette approche. Elle nous est aussi suggérée par la non-entité de l'Utopie, ce non-lieu dont la capitalefantôme est située au bord d'une rivière sans eau.

De plus, si nous posons le tableau de l'Utopie contre le tableau de l'histoire, l'Etat imaginaire contraste avec le système de représentations et les us et coutumes de l'Angleterre des Tudor, même si les analogies ne sont pas tout à fait absentes. Certains aspects sont empruntés au Moyen âge, mais la cité philosophique tient de l'Antiquité grecque et ses hommes, étant parvenus à développer une sagesse rationnelle, ont aussi la perfection des hommes de nature des récits de voyages au Nouveau Monde. Les Utopiens ne sont donc ni de l'Ancien ni du Nouveau Monde, sans être étrangers à l'un ou à l'autre. Ils amalgament de l'histoire certaines caractéristiques, tout en étant, par cet effet de distanciation de l'imaginaire, coupés du temps et de l'espace des hommes.

La césure fait d'ailleurs partie du récit. En conquérant Abraxa, le roi Utopus coupa l'isthme qui retenait la presqu'île au monde terrestre. Il créa une île radieuse, éloignée, protégée de la terre, et l'aménagea selon une architecture géométrique. Cette île est divisée en cinquante-quatre comtés et ses villes ont des plans identiques. Ses habitants parlent la même langue, obéissent au même lois et partagent les mêmes coutumes. Repliée sur ellemême, elle n'est pas victime du temps linéaire des hommes. En effet, depuis son origine, sa clôture protège son temps périodique et répétitif, sorte d'éternité que symbolisent les recommencements de la mer quil'entoure et en rend l'accès difficile.

A une époque caractérisée par le changement économique et social, les soulèvements et la guerre, l'éclatement des limites géographiques et la remise en question de l'autorité, Thomas More a imaginé un univers clos et statique où règnent l'égalité, l'amour de la paix et la simplicité de la vie commune. A la réalité sociale historique, Thomas More oppose une autre réalité, fictive, éloignée, à l'abri des actions ou des défis du temps. Mais dans leur rapport de différence, les Utopiens ne sont pas tout à fait étrangers aux hommes de la terre.

C'est en nous appliquant à retracer, dans la reconstruction fictive de l'Utopie, les liens que les Utopiens entretiennent avec le présent et le passé des hommes de la terre, que nous nous proposons, dans cet article, d'étudier les nouveaux hommes imaginés par Thomas More à la Renaissance, les principales caractéristiques de leur Etat, leurs coutumes sociales et domestiques ainsi que leurs idées et leurs croyances. 


\section{L'Etat}

Représentons-nous une île en forme de croissant de lune. Ses extrémités se ferment sur un détroit protégé par des récifs. Au milieu du détroit, se dresse une tour où des vigiles montent la garde. Les rives extérieures se découpent escarpées et rocheuses et, à certains endroits, de hautes clôtures empêchent d'accoster. Les rivières sont inconnues et inaccessibles aux étrangers. Cette île fantastique est donc refermée sur elle-même et protégée par une double clôture,c'est-à-dire par sa configuration géographique et par la main de ses hommes. Le passé de l'Utopie est archaïque et, lorsque Pierre Gilles met en doute que l'Etat de cette terre nouvelle soit meilleur que ceux des sociétés connues, Raphaël répond que, si on accorde foi à l'histoire de ce monde, "les villes existaient là-bas avant même qu'il y eût des hommes chez nous." 4

Les villes ont érigé leurs murs et leurs bastions dans chaque comté, comme si elles étaient uniques et se protégeaient des autres. Elles sont pourtant de même configuration et de même composition - il s'agit en effet d'en connaître une pour les connaître toutes ${ }^{5}$ - elles paraissent stables, ni objets, ni sujets des désirs d'expansion ou de domination. Afin que les Utopiens connaissent le travail de la terre, un échange des citadins et des paysans est prévu selon des cycles précis et ainsi les villes sont en constant rapport avec la campagne.

On peut voir dans le système agraire de l'Utopie une opposition à l'émergence en Angleterre d'une nouvelle économie basée depuis le XVe siècle sur l'élevage des moutons, l'industrie et le commerce de la laine. On peut y voir aussi un plaidoyer en faveur de la campagne à une époque où les terres deviennent propriétés de pâturage et où les villes acquièrent des richesses et tendent à consolider leur pouvoir politique. Il s'agirait, semble-t-il, d'une approbation de la part de Thomas More de l'ancien système d'agriculture intensive et de vaine pâture auquel correspondait le paysage d'openfield. 6

Il est clair en effet que s'affiche, dans la répartition judicieuse des terres arables entre les Cités, dans la primauté du métier d'agriculture pour les hommes et les femmes, dans la participation régulièrement renouvelée des personnes de la ville au travail agricole, dans la responsabilité des Philarques et des père et mère de famille, le recul que prend Thomas More par rapport à ce qu'on a appelé la révolution agraire du XVIe siècle qui, progressivement, s'est caractérisée, entre autres, par la croissance des fermes, la clôture des terres arables, le développement des pâturages au détriment des labours, l'exode des villageois vers les villes et l'émancipation du fermier du contrôle communal. ${ }^{7}$ D'ailleurs, la description qu'offre Thomas More, au Livre I, du déclin des communautés villageoises et de l'appauvrissement des fermes au profit de quelques propriétaires terriens ne cache pas la véhémence de sa critique. ${ }^{8}$ 
Le système socio-économique des Utopiens dans lequel, s'il n'y a pas de place pour la monnaie, par contre, personne ne manque du nécessaire, ${ }^{9}$ porte aussi une critique dans son contraste avec les conditions socioéconomiques réelles des Anglais du début du XVIe siècle. La hausse des prix qui affectait à la fois les loyers et la nourriture contribuait à la misère quotidienne d'un grand nombre d'habitants, alors que quelques gentilhommes, bourgeois acheteurs de terre, ou des gens de plus modeste origine, protégés par les faveurs royales, étendaient leur pouvoir sur le sol et tiraient leurs profits des coûts réduits de la production de la laine et de la nourriture, et de l'expansion commerciale. ${ }^{10}$ En Utopie, le bien comun politique permet de pourvoir aux besoins des personnes. Alors qu'il s'objecte au collectivisme au Livre I, parce que, dit-il, l'esprit communautaire s'édifie à partir du souci de la propriété privée, ${ }^{11}$ Thomas More inverse cette position dans sa fiction utopique: "ici, où rien n'est privé, on s'occupe sérieusement des affaires publiques." 12

Par son communisme, la société des Utopiens aurait réalisé à son plus haut point l'esprit chrétien de justice universelle. Elle se distingue des autres peuples comme se distinguaient dans leur milieu au Moyen âge les communautés de moines ou de frères vivant sous le régime de propriété commune. ${ }^{13}$ On sait que Thomas More partageait avec les humanistes l'idéal de la communauté de biens et on connaît l'influence des Frères de la vie commune, entre autres, sur les humanistes du Nord de l'Europe. ${ }^{14}$ Dans sa lettre à un moine qui parut en 1520, Thomas More rappelle que, selon le décret divin, toutes les choses doivent être partagées, et que le Christ a invité les hommes à abandonner la propriété privée pour la propriété universelle; il apparaît évident à l'humaniste que l'homme poursuit ses affaires privées au détriment de la propriété commune à cause de sa nature déchue. ${ }^{15}$

Dans l'Utopie, Thomas More prête à Raphaël le discours suivant: "Je ne puis douter que l'intérêt personnel de chacun et l'autorité du Christ, notre Sauveur, ... n'aurait déjà, depuis longtemps et avec beaucoup de facilité, amené le monde entier à adopter les lois de cette République. ..."16 Les Utopiens ne sont pourtant pas chrétiens. Quand il pense à la propriété, Raphaël retient la position de Platon: "Ce très grand sage avait prévu qu'il n'y avait pour la société qu'une seule et unique voie de salut: imposer l'égalité des biens."17 Mais, loin de reproduire la république antique, Thomas More a imaginé une société sans classe et il a étendu le communisme aristocratique de Platon à l'ensemble du peuple utopien. ${ }^{18}$

Raphaël aurait pu lui-même remarquer la ressemblance entre les Utopiens et les hommes du Nouveau Monde qu'il avait visités lors de ses voyages avec Vespucci. Comme les tribus indiennes, les Utopiens ne sont pas divisés en classes, ils partagent des habitations communautaires, ils méprisent l'or, ils ne vendent ni n'achètent et ils se contentent de ce que la 
nature leur offre. ${ }^{19}$ Mais la nécessité de la loi selon laquelle toutes les choses appartiennent à tous, pour que chacun jouisse de l'abondance, donne au plaisir antique des Utopiens l'austérité des communautés chrétiennes médiévales.

Pas plus qu'il n'est entièrement le système économique du XVIe siècle inversé, le système économique de la société égalitaire des Utopiens ne se conforme entièrement au modèle idéal grec ou à la pratique médiévale, à l'Ancien ou au Nouveau Monde. Hybride, il reste autre, toujours à distance.

La prospérité et le bonheur que le communisme apporte aux Utopiens sont protégés par la vigilance des gouvernants. Des études ont montré l'influence de la Politique d'Aristote et des commentaires médiévaux sur la pensée politique de l'Utopie. ${ }^{20}$ Si les Grecs à qui ils ressemblent ont cherché une forme stable de gouvernement qui les rende heureux, les Utopiens sont parvenus à réaliser un Etat où règne la justice, et Thomas More n'a pas non plus ignoré, pour son discours, certains principes de la République de Platon et de la République de Cicéron. ${ }^{21}$

Cependant, à cause du caractère démocratique de leurs institutions politiques, les Utopiens se situent dans la tradition anglo-saxonne. En effet, dans l'Etat utopien, nous trouvons les composantes majeures du pouvoir politique de l'Angleterre médiévale. Les fonctions publiques sont électives et le prince des Utopiens est élu, ${ }^{22}$ comme les premiers rois furent choisis, à la tête des tribus teutones unifiées. Il a besoin de l'appui de son Conseil pour gouverner, comme les rois du XIe siècle, représentant toujours le peuple, avaient besoin de l'Assemblée nationale, et il peut être déposé, si on le soupçonne de tyrannie, comme les rois de l'histoire ont été déposés par le Grand Conseil des Nations, au moins six fois, entre Sigeberht de Wessex et Jacques II. Les magistrats, qu'il s'agisse des Syphograntes élus par les familles ou des Protophylarques, leurs chefs, représentent le peuple au Conseil d'Etat, fonction analogue à celle des chevaliers du temps des rois Jean Sans Terre en Henri III. Le pouvoir politique est avant tout le pouvoir du peuple. En effet, avec la mobilité des députés, les Utopiens sont protégés contre la conspiration de leur Conseil qui pourrait chercher à les opprimer ou à modifier leur bien public et la consultation des familles pour les questions importantes leur reconnaît un droit à participer aux décisions de l'Etat. ${ }^{23}$ En Utopie, le pouvoir du prince repose donc sur le pouvoir du peuple comme, en Angleterre, le pouvoir de la couronne reposa d'abord sur les assemblées populaires, puis sur l'assemblée des députés. Et les droits du peuple sont protégés par la structure de l'Etat comme, au XIIIe siècle, la Grande Charte consolidait les droits du peuple contre la législation et la taxation arbitraire des rois.

Les Tudor maintinrent les institutions politiques médiévales mais les utilisèrent pour consolider le pouvoir monarchique au détriment de la seigneurie féodale. ${ }^{24}$ Dans une lettre datée du 25 juillet $1519,{ }^{25}$ Erasme dit 
que Thomas More avait l'intention de dépeindre l'origine des maux de l'Etat et qu'il modela l'Utopie sur la constitution britannique avec laquelle il était familier. Or, par son discours utopique, Thomas More effectue un glissement du présent au passé, vers une constitution non altérée par les motifs de l'histoire, de la pratique au modèle idéal, vers un Etat originel de justice. Et cet Etat reste autre, refermé sur lui-même, et sur lui règne un roi sans peuple.

\section{Les coutumes sociales et domestiques}

Quelques coutumes sociales et domestiques des Utopiens indiquent que Thomas More a donné en Utopie une nouvelle résidence au projet monastique qu'il retrouve à son point zéro, contre la dérive de l'histoire qui avait conduit les monastères à l'illégalité, l'immoralité et l'ignorance pendant le Moyen âge. On pourrait supposer que Thomas More se soit servi comme modèle de la Chartreuse de Londres où il avait séjourné quatre ans et qui, restée fidèle à sa règle, était un îlot de prière si bien ancré dans la tradition que, a-t-on dit, saint Bède ou saint Cuthbert auraient pu s'y retrouver sans étonnement. ${ }^{26}$

Les correspondances ne manquent pas. L'ordonnance des heures chez les Utopiens, le travail manuel obligatoire et la répartition des tâches sont conformes à la Règle de saint Benoit. ${ }^{27}$ Les simples habits, la réserve des conversations, la lecture quotidienne et l'atmosphère des églises rapellent les Coutumes de Guigues. ${ }^{28}$ Comme les Chartreux au chapître, les Utopiens se réunissent en conseil, délibèrent des questions communes et votent. ${ }^{29}$ Conformément à la Règle bénédictine et à la pratique monastique, ${ }^{30}$ les Utopiens avouent leurs fautes en famille et demandent pardon, non seulement pour se libérer la conscience, mais surtout pour se réconcilier. ${ }^{31}$

S'ils pensent que la contemplation de la nature est une façon de louer Dieu, les Utopiens mènent une vie active. Ils se tiennent volontiers occupés aux travaux collectifs les plus exigeants ou aux tâches serviles. ${ }^{32}$ L'oisiveté est bannie de l'Utopie parce qu'elle compromet la félicité future et les Syphograntes, dans leur vigilance à surveiller les occupations des citoyens, pourraient s'inspirer de la Règle monastique pour laquelle "l'inaction est l'ennemie de l'âme." ${ }^{33}$ On se demandera si Thomas More n'a pas réalisé en Utopie la réforme religieuse souhaitée depuis le XIVe siècle en y projetant l'esprit de la Devotio Moderna selon lequel, d'après l'auteur de l'Imitation de Jésus-Christ, "ce qui transforme véritablement l'homme religieux n'est pas l'habit ou la tonsure, mais le changement de manières et la parfaite mortification des passions." ${ }^{34}$ Les Utopiens ne possèdent rien, tout en n'étant privés d'aucune commodité, ils reconnaissent la sainteté de leur secte chaste et austère et, s'ils s'enfuient ou n'obéissent pas aux lois de la cité, ils sont sévèrement punis. ${ }^{35}$ Pauvres, chastes et obéissants, les Utopiens ressemblent à des moines. ${ }^{36}$ 
Cependant, l'analogie n'est pas complète et nous glissons d'un lieu à un autre, du cloître où se métamorphose l'âme du religieux à l'Utopie où l'équilibre des habitudes concourt au confort des hommes. Les monastères s'inscrivent dans le sacré. Derrière la clôture qui les sépare du monde, les moines naissent à une vie nouvelle et la vision que leur pratique oppose à la vie sociale existante prend un sens dans la médiation qu'ils assument entre le monde et Dieu. L'Utopie n'a pas d'eschatologie qui la transfigure. Elle ne connaît de relations qu'horizontales et reçoit de la simple nature les règles qui agencent le plaisir et la sagesse de ses hommes.

La clôture, l'organisation de la vie, l'autarcie ou l'obéissance sont autant de traits qui rapprochent l'Utopie et le monastère. ${ }^{37}$ Cependant, l'Utopie s'éloigne du projet monastique, non seulement parce qu'elle n'épouse pas sa vision religieuse, mais parce que, en off rant à l'humaniste une résidence sereine où il peut se tenir à l'abri des futilités accaparantes des princes, ${ }^{38}$ le discours utopique a, par déplacement, ajouté à cet autre lieu les traits de la philosophie antique.

\section{Les idées et les croyances}

Les espaces qu'occupent les idées et les croyances des Utopiens s'entrecoupent et se recouvrent, de telle sorte que nous trouvons dans l'interprétation humaniste ou la lecture médiéviste de l'Utopie des arguments complémentaires. D'une part, en donnant à ses nouveaux hommes une philosophie qui s'harmonise avec la religion naturelle, Thomas More a pu projeter en Utopie un équilibre moral qui contraste avec les vices de l'Europe du début du XVIe siècle ${ }^{39}$ et laisser soupçonner, par une didactique plus chrétienne que philosophique, selon l'éloge de Beatus Rhenanus, ${ }^{40}$ que la grâce peut conduire les chrétiens à un point de plus grande perfection que la nature n'a su conduire les païens. D'autre part, les vérités essentielles à la religion des Utopiens trouvant leurs étais dans la raison, on remarque en Utopie une continuité avec la fides quaerens intellectum de la tradition chrétienne médiévale. ${ }^{41}$

Dans leur philosophie, les Utopiens prennent les traits de l'Antiquité classique et, en cela, ils sont conformes à l'humanisme pour lequel la philosophie grecque de l'Antiquité constitua un apport des plus significatifs. L'affinité est sous-entendue dans le récit de l'acculturation des Utopiens et Raphaël va jusqu'à penser que ces insulaires tiennent leur commencement des Grecs. ${ }^{42}$ Avec leur religion naturelle déiste, les Utopiens ne sont pas en contradiction avec la religion chrétienne révélée et, même, plusieurs d'entre eux se sont convertis et ont été baptisés. ${ }^{43}$ Cependant, à cause d'un déplacement inhérent au discours utopique, les similitudes sont perturbées et la philosophie religieuse des Utopiens est autre, ni humaniste, ni chrétienne. 
Les Utopiens ont développé une philosophie morale qui ne sépare pas la félicité de l'homme de la vertu, la vertu du plaisir, le plaisir de l'accord avec la nature. La polarisation de la philosophie autour du plaisir et l'économie des plaisirs sur laquelle veillent les Utopiens témoignent de l'épicurisme du système de représentations en Utopie. Par ailleurs, la norme de moralité vient de Platon ${ }^{44}$ et les Utopiens placent au-dessus des délectations corporelles les plaisirs de l'esprit et, par-dessus tout, le plaisir de la conscience morale. ${ }^{45}$ De plus, à l'encontre d'Épicure qui avait supprimé la fabulation des dieux, les Utopiens cherchent dans les principes religieux de l'immortalité de l'âme, de la providence divine et des sanctions futures un contrefort à leur philosophie. Mais si la raison est en elle-même impuissante à trouver la vraie félicité et si elle fait appel à la croyance, par un jeu de renvoi, les principes essentiels de la religion reposent sur des preuves rationnelles. Bref, si la philosophie des Utopiens a besoin de la religion, leur religion naturelle se présente comme un éloge de la sagesse rationnelle.

Thomas More projette en Utopie une sagesse hédoniste en accord avec la philosophie d'Epicure qui reconnaît à la raison le pouvoir de mettre ses limites au corps. ${ }^{46}$ En plus, il y apporte par déplacement la double correction de la philosphie platonicienne et de la philosophie humaniste. Il glisse d'un lieu à l'autre, légitimant d'une part le plaisir et préférant d'autre part l'austérité ascétique. ${ }^{47}$

Pour ce qui est de la religion, l'Utopie semble offrir, au seuil de la Réforme, une résidence privilégiée au pluralisme et à la tolérance. Les cultes sont multiples et la loi voit à contenir le prosélytisme. Mais la plupart des Utopiens ont abandonné le polythéisme et croient à l'existence d'un être suprême, commencement et fin de toutes choses..$^{48}$ Thomas More a ainsi projeté dans la religion dominante des Utopiens la croyance en Dieu le Père, non seulement créateur mais providence de l'univers, avec le souci toutefois de retenir, comme dogmes essentiels, des vérités auxquelles étaient parvenus les anciens philosophes, Platon en particulier. ${ }^{49} \mathrm{Si}$ les Utopiens abandonnent leurs coutumes polythéistes, c'est qu'ils se laissent gouverner par la raison plus que par la peur supersticieuse; si, ayant entendu parler des enseignements du Christ, de ses préceptes et de ses miracles, ils sont disposés à le suivre, ${ }^{50}$ c'est qu'ils sont éminemment sages.

En Utopie, nous passons de la raison à la foi, pour mieux retrouver la raison. La religion des Utopiens, quoique conciliable avec la religion chrétienne, en est fondamentalement différente, parce qu'elle a son point d'attache dans la nature, ce qui explique que Raphaël suppose une secrète inspiration de Dieu qui ait conduit ces hommes-philosophes à se convertir. ${ }^{51}$ 
Retenons un autre détail significatif du récit de Raphaël. Après avoir été instruits de la religion chrétienne et avoir adhéré au Christ par le baptême, les Utopiens s'apprêtent à choisir parmi eux les prêtres qui dispenseront les sacrements. ${ }^{52}$ Dans son discours, Thomas More maintient un espace pour la religion privée et fait écran à l'universalisme de la religion chrétienne. En instituant leur propre Eglise, les Utopiens s'éloignent de la communauté spirituelle chrétienne. Ils ne sont donc plus chrétiens, ils sont autres, à distance.

\section{Conclusion}

Nous n'oublions pas que l'Utopie est d'accès difficile et notre étude confirme que toute proximité comporte un envers de distance. C'est donc sur l'effet de la distanciation que nous aimerions développer quelques considérations, nous demandant d'abord si elle n'est pas liée à un jeu de paradoxe.

Un premier jeu efface l'espace. Thomas More va de l'Angleterre à l'Europe, à la Grèce ou au Nouveau Monde, recouvrant les lieux et les niant dans un ailleurs non géographique. La fiction donne des contours à l'univers utopien et aucun point de son espace n'échappe à l'architecture qui le divise et l'organise. En projetant et en recomposant les lieux réels dans un lieu autre, Thomas More met en évidence l'espace réel qu'il nie dans son jeu. En passant parle lieu fictif de l'Utopie, on ne peut que mieux connaitre l'Angleterre du début du XVIe siècle. Mais il y a plus, Thomas More imagine un homme qui pourrait être de partout, quoiqu'il ne soit nulle part.

Au jeu de l'espace se superpose celui du temps. L'Utopie a un commencement, mais elle est sans futur, et ses transformations, obéissant à une mécanique interne, n'ont pas la direction que donne la finalité de la croissance ou du salut. Thomas More a projeté en Utopie des particularités de son temps et du passé, mais sans assurer la coïncidence avec le passé. Son rêve n'est pas non plus nostalgique comme l'est, par exemple, le rêve de l'Atlantide perdue. En recomposant la réalité présente avec la réalité passée et en l'ouvrant sur les promesses des récits de voyages de son époque, Thomas More a imaginé un nouvel homme qui est de tous les temps, sans être enraciné dans l'histoire, c'est-à-dire en n'étant d'aucun temps.

Le jeu se poursuit dans l'organisation sociale, économique et politique de l'Utopie. Il articule les échanges, le gouvernement ou les habitudes en les superposant au recouvrement des lieux. Les Utopiens ressemblent aux Grecs par leur communisme, ou aux Amérindiens, mais davantage aux chrétiens; leur politique est grecque dans sa philosophie, mais anglosaxonne dans ses institutions; leurs coutumes se modèlent en plusieurs 
points sur la vie monastique, sans qu'ils soient pour cela des moines. En Utopie, les citoyens ne peuvent dominer les autres ou résister à la domination, car ils n'ont aucune propriété. Ils ont perdu leur individualité dans la collectivité, leur volonté fait corps avec celle de l'Etat et leurs actions sont réglées jusque dans les détails de la vie quotidienne. En veillant à la cohésion de son royaume imaginaire, Thomas More a mis les Utopiens à l'abri des actions du temps et de la liberté. Dans la distance qui les sépare de la réalité sociale historique, il les a dépouillés de toute qualité distinctive. Il les a établis dans un monde de justice et d'égalité, où les lois sont immuables et où le nouveau citoyen peut se reconnaître s'il abandonne ce qui le différencie des autres hommes. ${ }^{53}$

La philosophie et la religion qui légitiment l'Etat utopien se superposant aux autres tableaux selon le même jeu de recouvrement, de position et de distanciation. La philosophie du plaisir est redressée par la vertu de la religion et la foi a son fondement dans la raison naturelle. Thomas More a veillé sur la frontière qui sépare le religieux du profane. A une époque où le christianisme cherchait à se purifier par un retour aux origines, il a projeté en Utopie une philosophie religieuse qui se modèle sur l'Antiquité païenne et imaginé des hommes que la nature a conduits à un degré de perfectionnement tel, que la croyance les approche du Dieu des chrétiens. Cependant, en prenant l'homme et non Dieu comme exemple, en oblitérant le sens que donne la révélation à l'aventure humaine, Thomas More substitua à la religion une sagesse qui trouve sa mesure en elle-même et, à l'amour, la nécessité de la loi et l'harmonie de la nature.

L'Utopie reste donc toujours à distance, en rupture. Par les jeux qu'il déploie, le rêve utopique s'approche et s'éloigne des lieux de l'histoire, sans parvenir à compléter la description de son propre lieu. Les contours de l'île merveilleuse et inaccessible sont définis dans le récit et sa perfection semble immuable, mais sa qualité première est d'être autre et le discours reste en-deça de la définition de sa perfection. L'Utopie surpasse en excellence la cité philosophique de Platon, dit le poète Anemolius,${ }^{54}$ mais son excellence est fictive et elle-même inachevée.

Ceci apparaît plus clairement quand Thomas More entretient Erasme de son rêve dans level les Utopiens l'ont choisi pour être leur roi. Thomas More, qui reconnaît la sagesse de ses nouveaux hommes, est à son tour reconnu par eux. La sagesse des Utopiens prend alors un sens. Mais, en plus, puisque le rôle du roi est de conduire son peuple au bien, la destinée des hommes-philosophes que Thomas More a situés hors du temps et de l'espace se réalise par la médiation d'un être de l'histoire, Thomas More lui-même, humaniste anglais du début du XVIe siècle. Et le jeu se poursuit, dans la réalité, avec les allusions critiques qu'il porte; en effet, Thomas 
More invite son ami Erasme à le visiter en Utopie et il lui promet que les Utopiens lui rendront tous les hommages qui lui reviennent. 55

\section{Université de Montréal}

\section{Notes}

1 P.S. Allen, Opus Epistolarum Des, Erasmi Roterdami vol. 1-12 (Oxford: Clarendon, 1906-1963), tome II, Lettre 499, p. 414.

2 L'affirmation d'une distance caractéristique du discours utopique a déjà été mise en valeur par Louis Marin dans "De l'Utopia de More à la Scandza de Cassiodore-Jordanès", Annales, Economies, Sociétés, Civilisations, 26 (1971), 319, ainsi que ses aspects de projection et de déplacement, dans Utopiques: jeux d'espaces (Paris: Minuit, 1973), p. 249.

3 Nicholas Harpsfield, "The Life and Death of Sir Thomas More, Knight, Sometime Lord High Chancellor of England," dans Lives of Saint Thomas More, éd. E.E. Reynolds (London: Dent, 1963), p. 110.

4 Thomas More, L'Utopie, éd. A. Prévost (Paris: Mame, 1978), I, 68.

5 L'Utopie, II, 74.

6 H.W. Donner, Introduction to Utopia (London: Sidgwick \& Jackson, 1945), p. 57.

7 Cf. E. Lipson, The Economic History of England (London: Adam and Charles Black, 1949), I, 133-184; J.H. Hexter, Reappraisals in History (Aberdeen: Northwestern University Press, 1962), p. 83; P. Williams, Life in Tudor England (London: B.T. Batsford Ltd., 1964), p. 23-45.

8 L'Utopie, II, 39-42.

9 Ibid, p. 159-160.

10 Cf. P. Williams, Op. cit; F. Braudel, Les jeux de l'échange (Paris: Armand Colin, 1979), II, 421.

11 L'Utopie, I, 67.

12 L'Utopie, II, 156.

13 Cf. H.W. Donner, Op. cit, p. 74.

14 E.L. Surtz, The Praise of Pleasure (Cambridge: Harvard University Press, 1957), p. 164-165, 180.

15 E.F. Rogers, éd., St. Thomas More: Selected Letters (New Haven: Yale University Press, 1961), p. 85.

16 L'Utopie, II, 160.

17 L'Utopie, I, 65.

18 Lois,V, 741b - 743c; République, IV, 419a - 420b; Timée, $18 \mathrm{~b}$.

19 Cf. A.L. Morton, The English Utopia (London: Lawrence \& Wishart), 1969.

20 Cf. T.I. White, "Aristotle and Utopia," RQ, 29, (1976) 635-675; P.A. Duhamel, "Medievalism of More's Utopia," SP, 52, (1955), 99-126.

21 Cicéron définit l'Etat comme appartenant au peuple (République, I, XXV, 30). Pour Platon, la justice est incarnée dans l'Etat (République, II, 368e; IV, 427e). Cf. John Ferguson, Utopias of the Classical World (London: Thomas \& Hudson, 1975), p. 186.

22 L'Utopie, II, 77-78.

23 Ibid, p. 78.

24 Cf. R.B. Smith, Land and Politics in the England of Henry VII(Oxford:Clarendon Press, 1970), p. 254-262.

25 Lettre à Ulrich Hutten, dans P.S. Allen, Op. cit., tome IV, Lettre 999, p. 21.

26 J.A. Froude, History of England (London: Parker, Son and Bourn, 1862), II, 364.

27 L'Utopie, II, 80-81; Migne, PL., LXVI, 703-704; Cf. P.A. Duhamel, Op. cit., p. 119. 
28 L'Utopie, II, 85, 91, 92, 152; Migne, PL., CL111, 651-652, 693-694, 712-713, 727-728, 737-738.

29 L'Utopie, II, p. 78; Migne, PL., CL111, 661-662.

30 Migne, PL, LXVI, 501-502; voir aussi E.L. Surtz, The Praise of Wisdom (Chicago: Loyola University Press, 1957), p. 198-199.

31 L'Utopie, II, 153. A. Prévost note que, au temps de Thomas More, cet usage de la confession et de la réconciliation fraternelle était observé dans les familles et parmi les religieux (p. 153, no 1).

32 L'Utopie, II, 147-148.

33 Ibid., 80, 147; Migne, PL., LXVI, 703.

34 E.L. Surtz, The Praise of Wisdom, p. 110.

35 L'Utopie, II, 87-93, 148.

36 Quelques analyses récentes font ressortir cet aspect. Entre autres A. Prévost, L'Utopie, p. XV, parle de l'empathie profonde entre la vie cénobitique et l'Utopie. Voici ce que disent F.E. Manuel et F.P. Manuel, dans Utopian Thought in the Western World (Cambridge, Mass.: The Belknap Press, 1979), p. 123: "When compared with the modes and institutions of contemporary English society, Utopian life was indubitably nearer to Christian truth." F. Alistair, pour sa part, dans Thomas More: History and providence (Oxford: Basil Blackwell, 1982), p. 56, affirme ceci: "... More enviously viewed the Utopian as successful Carthusian (Franciscan if you like) mercers, the governing of whom would allow him to bring the cloister into court."

37 Cf. G. Lapouge, Utopie de civilisation (Genève: Librairie Weber, 1973), p. 64-69.

38 Lettre de Thomas More à Erasme, octobre 1517, dans P.S. Allen, Op. cit., tome III, Lettre 688, p. 111: "Tuum consilium probo, qui non vis principum negociosis nugis implicari."

39 R.W. Chambers, Thomas More (London: Jonathan Cape, 1935), p. 127.

40 Beatus Rhenanus, préface des Epigrammes de Thomas More, 1518, dans L. Bradner et C.A. Lynch, The Latin Epigrams of Thomas More (Chicago: The University of Chicago Press, 1953), p. 5. Au sujet de l'interprétation humaniste de l'Utopie, voir E.L. Surtz, The Praise of Wisdom, p. 613. De plus, étant donné la connaissance que Thomas More avait de De Civitate Dei de saint Augustin, les notes critiques de A. Prévost, dans L'Utopie, p. xvii-xviii, donnent un point de vue fort pertinent: Dans la construction de la cité divine, Thomas More ajoute des plans entre la nature sauvage du peuple de la cité terrestre et la cité renouvelée par le christianisme. Les Utopiens, hommes de nature, ont une pré-disposition à recevoir l'héritage judéo-chrétien.

41 P.A. Duhamel, Op. cit., p. 103-109.

42 L'Utopie, II, 116.

43 Ibid., p. 142.

44 République, IV, 443d, IX, 591c-d.

45 L'Utopie, II, 112.

46 Epicure, Maxime 20.

47 L'Utopie, II, 148.

48 Ibid., p. 141.

49 Ménon, 81a-b; Phédon, 71c-72a, 78b-c, 80a-81a, 105e-107, 108a-c; République, I, 330d - 331b; $\mathrm{X}, 608 \mathrm{c}-611 \mathrm{a} ;$ Timée, $29 \mathrm{e}-30 \mathrm{~b}$.

50 L'Utopie, II, 142.

51 Ibid.

52 Ibid.

53 T. Molnar souligne l'absence de distinction dans l'Utopie, cf. L'Utopie, Eternelle hérésie, Paris, Beauchesne, 1973, p. 134.

54 Utopie, pour mon isolement par les anciens nommée,

Emule à présent de la platonicienne cité,

Sur elle, peut-être l'emportant - car, ce qu'avec des lettres

Elle dessina, moi seule je l'ai montré

Avec des hommes, des ressources et d'excellentes lois-

Eutopie, à bon droit, c'est le nom qu'on me doit.

Sizain d'Anemolius, dans l'Utopie, p. 11.

55 P.S. Allen, Op. cit., tome II, Lettre 499, p. 414. 